\title{
Primary Ewing's Sarcoma of Lumbar spine treated with Neo-adjuvant Chemotherapy and Surgery- A Case Report
}

\author{
Rohit A Thaker ${ }^{1,2}$, Himanshu Dodiya ${ }^{2}$, Shrikant Dhanani ${ }^{2}$, Ankur Patel $^{2,3}$
}

\section{Abstract}

Primary involvement of mobile spine compared to non-mobile spine is very less in case of Ewing's sarcoma (ES). There are no fixed guidelines for these types of tumors because of their low incidence. These tumors usually have very high sensitivity to chemotherapy and radiotherapy. Goal for the management of ES of the spine is adequate local control through complete removal of tumor by doing aggressive resection along with restoration of spinal stability and preservation of neurology. En bloc spondylectomy or extralesional resection with wide disease-free margin provides good oncological results with a longer survival. Whenever it is possible to give neoadjuvant chemotherapy, it is always better because it helps to shrink tumor, treat micrometastasis, and make surgical excision easier with wide margin resection. However, in some case of spinal ES, it may not be possible because of neurological compromise and they might have to be addressed first by surgery and neurological decompression. We report here one such cases of primary ES of mobile lumbar spine treated with neoadjuvant chemotherapy and then with en bloc excision of tumor. The clinical picture and imaging characteristics of patient were analyzed as well as the management modalities and outcome has been discussed.

Keywords: Ewing's sarcoma; Spine surgery; Neoadjuvant chemotherapy; Lumbar spine.

\section{Introduction}

The most common primary sites of involvement of Ewing's sarcoma (ES) are the extremities and pelvis [1]. ES is the second most common primary sarcoma of bone in children and adolescents [2]. Primary malignant sarcomas of the spine usually rare. The division of primary spinal ES into non-sacral and sacral is important and is important because of different behavior of ES in these two regions in terms of response to therapy and survival rates [3]. Most studies on ES involving the mobile spine are limited to case reports.

Whenever possible, adequate treatment of any primary malignant spine tumors is aimed at cure rather than palliation. The definitive management of ES of the spine, as in other locations, could include three main modalities: Surgery, multiagent chemotherapy, and radiotherapy. Treatment of the local disease such as in this case is primarily through initial

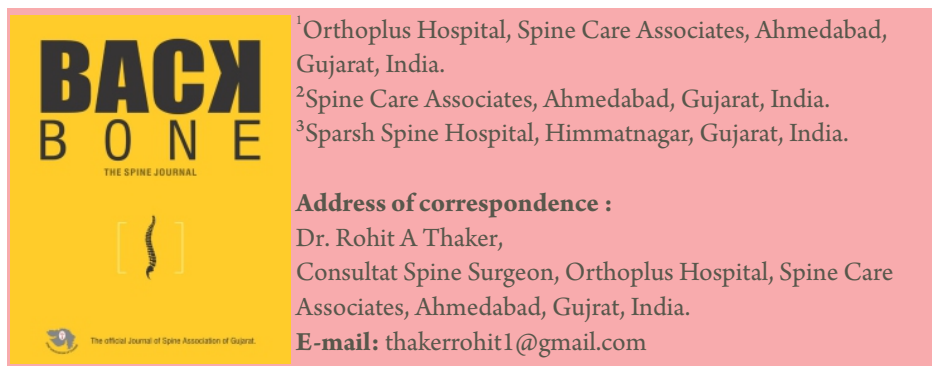

neoadjuvant or induction chemotherapy followed by local treatment including surgery and/or radiation [4]. Multiagent chemotherapy and multidisciplinary team approach has improved survival rate up to $65-80 \%$ in localized ES $[5,6]$, although overall survival (OS) remains dismal for patients with metastatic disease [7]. Management of ES usually starts with establishment of diagnosis by biopsy and then neoadjuvant chemotherapy of 3-6 doses is given, followed by surgical excision with wide clear margin/radiotherapy and then adjuvant chemotherapy [6]. However, management of primary spinal ES is little bit different when there is acute neurological deficit. Primary decompressive surgery has often been preferred to prevent major neurological sequelae [8]. However, a few recent studies reported that adequate and quick decompression of neural structures could be achieved by initial chemotherapy because of the high chemosensitivity of the tumor [4]. There is no consensus on the optimal form of local treatment for ES also as surgery and radiotherapy has their limits and associated with complications. Wide resection is often not feasible because of adjacent critical structures, while radiotherapy is limited as it is poorly tolerated by spinal cord [9]. Here, we present one such cases of mobile lumbar spine having primary ES treated with neoadjuvant chemotherapy and then surgical excision done with wide margin. 


\section{Case History}

A 19-year-old male patient presented to our clinic with progressively increasing low back pain associated with bilateral gluteal region pain and radicular pain for the past 4 months. The patient had developed paresthesia and tingling in right lower limb and he was walking with forward stooping gait. The patient had more of claudication type symptoms rather than severe low back pain. Visual analog scale (VAS) score at the first presentation was 5. On examination, tenderness was present in lumbosacral region and a movement of spine was terminally painful. Bilateral straight leg raising test was positive at around 30 . Neurological examination revealed right extensor hallucis longus/extensor digitorum longus (EHL/EDL) weakness of Grade 3/5 medical research council scale and hypoesthesia in L5-S1 dermatome. Rest neurological examination was normal. Bowl/bladder was intact. He was having a low-grade fever. Blood investigations revealed an elevated erythrocyte sedimentation rate, $36 \mathrm{~mm} / 2 \mathrm{nd} \mathrm{h}$, and C-reactive protein level, $14 \mathrm{mg} / \mathrm{L}$. The patient was further investigated in the form of Xray LS (lumbosacral) spine and magnetic resonance imaging (MRI) of LS spine with whole spine screening. X-ray was suggestive of lytic expansile lesion in posterior elements of L5 vertebrae. MRI revealed a large, enhancing expansile lesion involving bilateral L5 lamina, spinous process, and inferior articular process of L5 vertebrae with adjacent soft-tissue component showing intraspinal extension causing marked compression over cauda equina (Fig. 1). Whole body bone
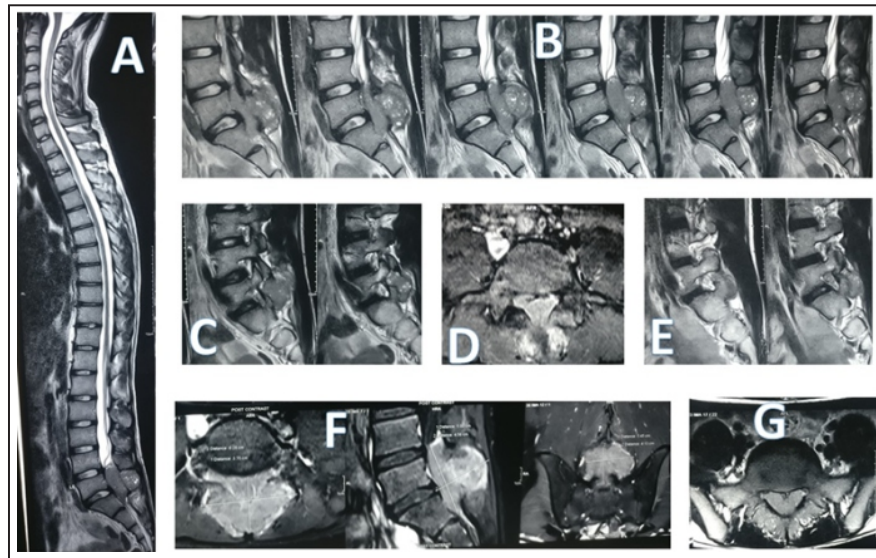

Figure 1: Magnetic resonance imaging showing large expansile lesion involving bilateral L5 lamina, spinous process, and inferior articular process of L5 vertebrae with adjacent soft-tissue component showing intraspinal extension causing compression over cauda equina. (A) Whole spine screening showing lesion in posterior elements of L5. (B) Sagittal T2 images showing significant soft-tissue extension inside spinal canal causing compression on thecal sac. (C-E) Extension of lesion in both inferior articular process of L5 but bilateral L5 pedicles was free. (F) Contrast MRI shows extension of tumor in all three planes. (G) Bilateral S1 pedicle disease free. scan study was done and it was negative for metastatic lesion of the disease. CT-guided biopsy (Fig. 2) was performed by interventional radiologist from L5 posterior elements after discussing with surgeon for possible future surgical approach and route for biopsy was taken in posterior midline so it can facilitate excision of biopsy tract in future. Material obtained by biopsy was sent for histopathological examination and it was suggestive of round cell tumor and immunohistochemistry was positive for CD99 and FLI1. The morphological and immunohistochemical profile was indicative of ES. The patient was started on neoadjuvant combination chemotherapy and was regularly followed up. The agents and doses used for combination chemotherapy for the patient were decide by oncologist and it included vincristine, doxorubicin, and cyclophosphamide alternating with etoposide and ifosfamide every 3 weeks. The patient was regularly followed up with

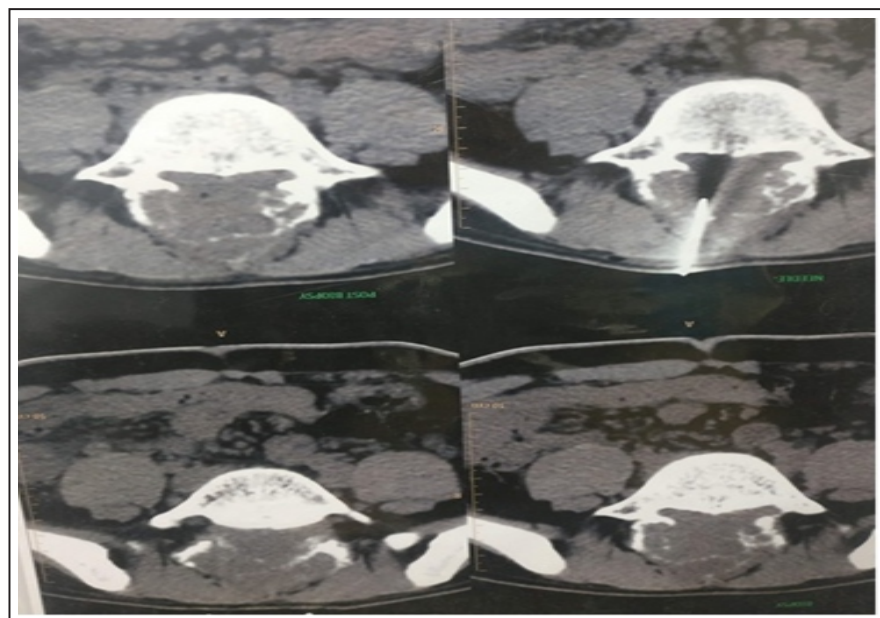

Figure 2: CT-guided biopsy from posterior elements of L5 done by radiologist. Image showing sequential CT cuts and position of biopsy needle in tumor mass.

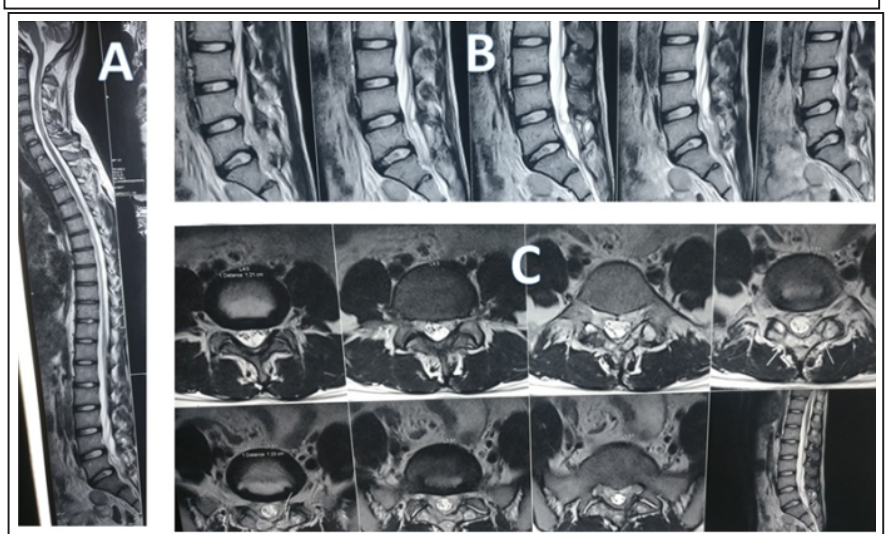

Figure 3: Magnetic resonance imaging study after chemotherapy. (A) Whole spine screening shows involvement of $L 5$ posterior elements. (B) Sagittal T2 images show complete clearance of soft-tissue lesion from spinal canal and no significant compression on thecal sac. (C) Axial images show that lesion was contained in bilateral lamina, spinous process, and inferior articular process of L5. 
oncologist during whole period of chemotherapy. After four cycle of chemotherapy, repeat MRI was done and it was suggestive of significant regression of tumor tissue. (Fig. 3) Changes of tumor were present in bilateral laminae, spinous process, and inferior articular process of L5. All the soft-tissue components compressing thecal sac were reduced. The patient also had significant improvement clinically, his claudication symptoms were reduced and back pain was also improved. VAS score at this stage was 3 . Neurologically, he was having Grade 3 power in the right EHL/EDL and hypoesthesia in L5-S1 dermatome. At this stage, we decided to operate and the patient was planned for surgery. The patient was positioned prone under general anesthesia. Standard posterior midline approach was taken with excision of biopsy tract. En bloc laminectomy of L5 laminae with spinous process and inferior articular process was done along with biopsy tract. (Fig. 4) As both inferior articular processes were removed and part of bilateral L5 pedicle was also removed to ensure wide margin, so to maintain stability of spine, L4-S1 pedicle screw fixation was done and intertransverse fusion was done using autograft. L5 screw was avoided as pedicle tract was open directly in spinal canal. Intraoperatively, dura was found adherent to laminae. Specimen was sent for histopathological examination and it shows wide margin, so complete excision of disease was confirmed. Post-operative period was uneventful and the patient was advised complete bed rest till suture removal was done. After that, the patient was mobilized with lumbosacral corset support. The patient was started on chemotherapy again after suture removal. The patient was regularly followed up. Total 9 cycles of chemotherapy were completed. At the end of chemotherapy, bone scan was performed again and it was suggestive of clearance of disease. At 2-year follow-up, the patient is disease free and relieved of all symptoms. X-ray taken at 2-year follow-up is suggestive of implants well in position (Fig. 5).

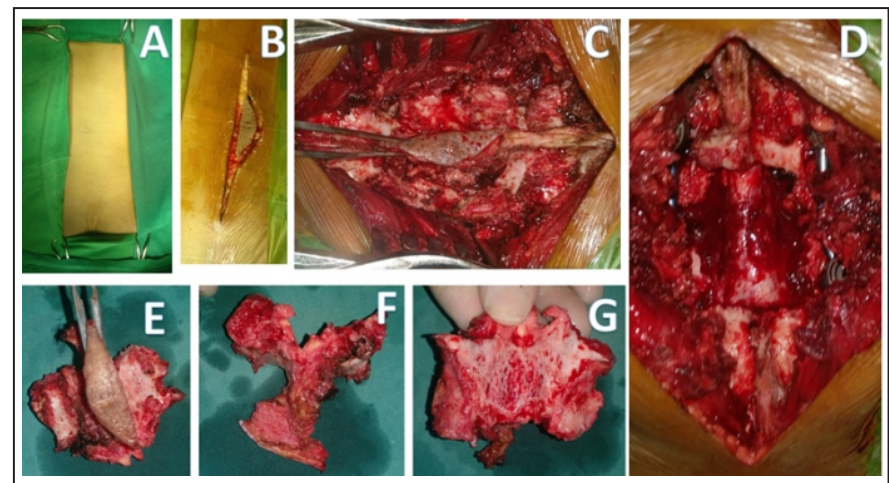

Figure 4: Intraoperative images. (A) Standard posterior midline approach. (B) Excision of biopsy tract. (C) Complete exposure with separation of biopsy tract. (D) En bloc laminectomy of L5 with biopsy tract and inferior articular process of L5. (E-G) Complete removal of tumor tissue.

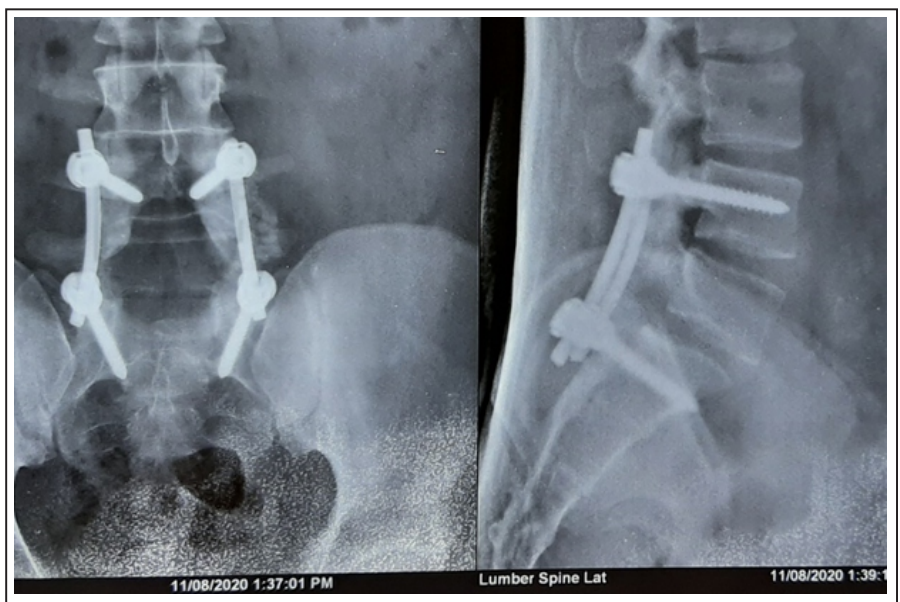

Figure 5: X-ray of lumbosacral spine anteroposterior and latera view at 2 -year follow-up showing implants well in position.

\section{Discussion}

ES of the spine is a rare condition that appears with a clinical triad of local pain, neurological deficit, and a palpable mass. The age at presentation ranged from 12 to 24 years. The first symptom is usually local back pain. When the patient presents in the first two decades of life and symptoms like severe local pain which are not relieved by conservative management and significant overlying tenderness/neurological involvement is present, physician should have high suspicion of possibility of spinal malignancy. Signs of ES are usually late to appear on radiograph and they usually appear after the neurological signs. The most common finding in X-ray is lytic bone destruction involving the vertebra [4]. The lytic changes varied from focal lytic lesion, which is more common, to complete flattening of the vertebral body (vertebra plana) [10]. Rarely, sclerotic changes can also be observed. CT scan is helpful in determining the extent of involvement of vertebral bodies and the posterior elements as well as in outlining the soft-tissue component. CT-guided needle biopsy of vertebral or paravertebral lesions can be accomplished with accuracy and relative ease and it helps in establishing diagnosis. MRI is better for visualizing epidural compression and tumor spread to the bone marrow or extension into adjacent soft tissues and also to assess relationship of tumor to adjacent vasculatures. Gadolinium-enhanced MRI showed the highest accuracy in determining the response to pre-operative chemotherapy [11]. MRI is also helpful in pre-operative planning to decide adequate surgical margins of the tumor, which can permit safer and more complete resection. ES is frequently multifocal. Skeletal scintigraphy (bone scan) before staging is an important step to rule out other foci and also it has been used in the follow-up treatment of primary ES [12]. On histopathological examination, ES presents as small round cell tumor. Differential diagnosis of a small round-cell bone tumor includes ES, neuroblastoma, primitive neuroectodermal tumor of bone, malignant lymphoma, and 
rhabdomyosarcoma. The differentiation between these tumors on basis of light histological data alone may not always be accurate, so in addition to light microscopy, electron microscopy, immunohistochemical, and occasionally cytogenetic analysis is current standard practice [13].

It is controversial whether the same treatment strategy of ES outside of the spine can be used for ES originating in the spine, especially when the patients present with major neurological deficits [7]. The timing of local treatment for ES of the spine is critical because spinal cord compression is a common emergency due to epidural tumor extension compressing neural tissue $[4,9,14]$. Venkateswaran et al. reported in their study series of 33 patients that neurological deficits were determined in $94 \%$ of all ES patients [14]. In study by Vogin et al., $79 \%$ of the 75 patients with ES of the spine presented with symptomatic neurological compression [9]. The degree of neurological recovery is often fixed at 48-72 $\mathrm{h}$ after spinal cord injury so in such cases, prompt decompression is urgently required because delay in treatment can result in irreversible outcomes [15]. In such circumstances, immediate surgical decompression is often preferred $[8,9]$. Of course, neurological outcome is important measure of treatment of spinal ES, but OS is also an important issue which needs to be addressed. Surgery as the first-line treatment of ES of the spine is controversial because it may induce local tumor spilling and ultimately compromise survival [16]. Neoadjuvant chemotherapy can eradicate micro metastases and induce shrinkage of the primary tumor, which contribute to effective local and systemic control $[4,17]$. In a study by Zhang et al., effects of chemotherapy on neu $\neg$ rological preservation in ES of the spine were reported [18]. The most likely explanation for this effect of chemotherapy is that the high chemosensitivity of the tumor leads to shrinkage of the tumor and then decreases compression of the spinal cord in a timely manner. As ES of spine is rare, there has been no comparison study has been made between these two types of first-line treatments. In a study by Zhang et al., they found that patients who had initial chemotherapy had significantly better event-free survival (EFS) and OS compared with patients who underwent primary surgery. Furthermore, induction chemotherapy contributed to a higher rate of R0 (microscopic negative margin) resection, which was a favorable prognostic factor of OS [18]. ES arising from sacrum and in older age group has poor prognosis and patients with metastatic diseases are always associated with poorer survival compared with patients with localized ES $[19,20]$. Hence, it is recommend performing surgery after induction chemotherapy instead of before chemotherapy to facilitate R0 (microscopic negative margin) resection and decrease surgical morbidity and risks [18]. Adequate spinal reconstruction and stabilization should be done to prevent subsequent development of spinal deformity and neurologi $\urcorner$ cal demise after performing en bloc resection. It is also important to emphasize that induction chemotherapy in patients who are neurologically at risk should start as early as possible once histopathological diagnosis has been established. In the case where a defini $\neg$ tive diagnosis is delayed (e.g., a soft-tissue mass within the vertebral canal without any bony lesion), emergency surgery should be con $\neg$ sidered as first-line treatment. This is because any delay in intervention would compromise effectiveness in alleviating a neurological deficit [4].

\section{Conclusion}

Chemotherapy as the first-line treatment of ES of the spine can achieve similar results as primary surgery in preserving neurological function, even in case of major neurological deficits. Initial chemotherapy is associated with better EFS and OS compared with primary surgery. Furthermore, induction chemotherapy contributes to a higher rate of en bloc resection with clear wide margin, which is an independent prognostic factor of improved OS.18 R0 (microscopic negative margin) resection following induction chemotherapy is strongly recommended whenever technically feasible. 


\section{References}

1. Berger M, Fagioli F, Abate M, Riccardi R, Prete A, Cozza R, et al. Unusual sites of Ewing Sarcoma (ES): A retrospective multicenter 30-year experience of the Italian Association of Pediatric Hematology and Oncology (AIEOP) and Italian Sarcoma Group (ISG).EurJ Cancer 2013;49:3658-65.

2. Gupta AA, Pappo A, Saunders N, Hopyan S, Ferguson P, Wunder J, et al. Clinical outcome of children and adults with localized Ewing sarcoma: Impact of chemotherapy dose and timing of local therapy. Cancer 2010;116:3189-94.

3. Pilepich MV, Vietti TJ, Nesbit ME, Tefft M, Kissane J, Burgert O, et al. Ewing's sarcoma of the vertebral column. Int J Radiat Oncol Biol Phys 1981;7:27-31.

4. Mirzaei L, Kaal SE, Schreuder HW, Bartels RH. The neurological compromised spine due to Ewing sarcoma. What first: Surgery or chemotherapy? Therapy, survival, and neurological outcome of 15 cases with primary Ewing sarcoma of the vertebral column. Neurosurgery 2015;77:718-24.

5. Marina NM, Pappo AS, Parham DM, Cain AM, Rao BN, Poquette CA, et al. Chemotherapy dose-intensification for pediatric patients with Ewing's family of tumors and desmoplastic small round-cell tumors: A feasibility study at St. Jude Children's Research Hospital.J Clin Oncol 1999; 17:180-90.

6. Ferrari S, Palmerini E, Alberghini M, Staals E, Mercuri M, Barbieri E, et al. Vincristine, doxorubicin, cyclophosfamide, actinomycin $D$, ifosfamide, and etoposide in adult and pediatric patients with nonmetastatic Ewing sarcoma. Final results of a monoinstitutional study. Tumori 2010;96:213-8.

7. Gaspar N, Hawkins DS, Dirksen U, Lewis IJ, Ferrari S, Le Deley MC, et al. Ewing sarcoma: Current management and future approaches through collaboration.J Clin Oncol 2015;33:3036-46.

8. Gopalakrishnan CV, Shrivastava A, Easwer HV, Nair S. Primary Ewing's sarcoma of the spine presenting as acute paraplegia.J Pediatr Neurosci 2012; 7:64-6.

9. Vogin G, Helfre S, Glorion C, Mosseri V, Mascard E, Oberlin O, et al. Local control and sequelae in localised Ewing tumours of the spine: A French retrospective study. EurJCancer 2013; 49:1314-23.

10. Papagelopoulos PJ, Currier BL, Galanis E, Grubb MJ, Pritchard DJ, Ebersold MJ.
Vertebra plana caused by primary Ewing sarcoma: Case report and review of the literature. J Spinal Disord Tech 2002;15:252-7.

11. Erlemann R, Sciuk J, Bosse A, Ritter J, Kusnierz-Glaz CR. Response of osteosarcoma and Ewing sarcoma to preoperative chemotherapy: Assessment with dynamic and static MR imaging and skeletal scintigraphy. Radiology 1990;175:791-6.

12. Estes DN, Magill HL, Thompson EI, Hayes FA. Primary Ewing sarcoma: Followup with Ga-67 scintigraphy. Radiology 1990;177:449-53.

13. Schmidt D, Harms D, Pilon VA. Small-cell pediatric tumors: Histology, immunohistochemistry, and electron microscopy. Clin Lab Med 1987;7:63-89.

14. Venkateswaran L, Rodriguez-Galindo C, Merchant TE, Poquette CA, Rao BN, Pappo AS. Primary Ewing tumor of the vertebrae: Clinical characteristics, prognosticfactors, and outcome. Med Pediatr Oncol 2001;37:30-5.

15. O'Phelan KH, Bunney EB, Weingart SD, Smith WS. Emergency neurological life support: Spinal cord compression (SCC). Neurocrit Care 2012;17:S96-101.

16. Boriani S, Amendola L, Corghi A, Cappuccio M, Bandiera S, Ferrari S, et al. Ewing's sarcoma of the mobile spine. Eur Rev Med Pharmacol Sci 2011;15:8319.

17. Subbiah V, Anderson P, Lazar AJ, Burdett E, Raymond K, Ludwig JA. Ewing's sarcoma: Standard and experimental treatment options. Curr Treat Options Oncol 2009; 10:126-40.

18. Zhang J, Huang Y, Lu J, He A, Zhou Y, Hu H, et al. Impact offirst-line treatment on outcomes of Ewing sarcoma of the spine. Am J Cancer Res 2018;8:1262-72.

19. Arshi A, Sharim J, Park DY, Park HY, Yazdanshenas H, Bernthal NM, et al. Prognostic determinants and treatment outcomes analysis of osteosarcoma and Ewing sarcoma of the spine. Spine J2017;17:645-55.

20. Uyeturk U, Helvaci K, Demirci A, Sonmez OU, Turker I, Afsar CU, et al. Clinical outcomes and prognostic factors of adult's Ewing sarcoma family of tumors: Single center experience. Contemp Oncol (Pozn) 2016;20:141-6.
Conflict of Interest: NIL Source of Support: NIL
How to Cite this Article

Thaker RA, Dodiya H, Dhanani S, Patel A | Primary Ewing's Sarcoma of Lumbar Spine Treated with Neoadjuvant Chemotherapy and Surgery - A Case Report| Back Bone: The Spine Journal October 2020-March 2021; 1(1):28-32. 